Check for updates

Cite this: RSC Adv., 2017, 7, 40362

Received 11th May 2017

Accepted 4th August 2017

DOI: 10.1039/c7ra05347a

rsc.li/rsc-advances

\section{Hydrophobic tagging-mediated degradation of Alzheimer's disease related Tau $\uparrow$}

\author{
$\mathrm{Na}$ Gao, ${ }^{a}$ Ting-Ting Chu, ${ }^{a}$ Qian-Qian Li, ${ }^{a}$ Yeh-Jun Lim, ${ }^{a}$ Tian Qiu, ${ }^{a}$ Meng-Rong Ma, ${ }^{a}$ \\ Zhi-Wen Hu, ${ }^{a}$ Xi-Fei Yang, ${ }^{c}$ Yong-Xiang Chen, ${ }^{\text {a }}$ Yu-Fen Zhao (D) a \\ and Yan-Mei Li (D)*ab
}

Tau reduction is a favorable strategy for Alzheimer's disease (AD) treatment. In this work, we designed and synthesized a hydrophobic tag conjugated peptide (HyT-Tau-CPP) to selectively promote the degradation of Tau. HyT-Tau-CPP could potently lower the Tau level in a concentration- and time-dependent manner. Furthermore, HyT-Tau-CPP could also reduce the level of Tau in the brain of AD mouse models. Therefore, Tau reduction by HyT-Tau-CPP may become a potential therapeutic strategy in the treatment for AD.
Alzheimer's disease (AD) is a devastating neurodegenerative disease. There is a lack of effective treatments for $\mathrm{AD}$, thus it is essential to develop a feasible strategy for its treatment. The microtubule-associated protein, Tau, plays a crucial role in $\mathrm{AD} .{ }^{1}$ Neurofibrillary tangles (NFTs) formed by Tau aggregates is one of the major hallmarks on the pathogenesis of AD. Tau dysfunction can cause the defect of microtubule function and even neuron loss. ${ }^{2}$ In addition, Tau can mediate amyloid- $\beta(A \beta)$ induced neurodegeneration. ${ }^{3}$ Recently, therapeutics targeting Tau have attracted much attention. ${ }^{4}$

Previous studies showed that the total amount of Tau was notably elevated in $\mathrm{AD}$ patients' brains, which may be toxic to neurons. ${ }^{5}$ Tau depletion could reduce Tau aggregation. Moreover, it has emerged that Tau level reduction could mitigate $A \beta$ toxicity, protect against memory impairment and improve survival in $\mathrm{AD}$ mouse models. ${ }^{3 b, c, 6}$ Therefore, lowering the level of Tau may be an effective strategy for treating AD.

Tau is a non-enzyme protein whose conformation is highly flexible without active pockets. ${ }^{7}$ There are currently two main strategies for the regulation of non-enzyme proteins. One strategy is genetic knockdown, which has distinct therapeutic potential. However, it has a few disadvantages such as poor safety and poor metabolic stability. ${ }^{8}$ The other strategy is chemical knockdown. ${ }^{9}$ Most of the chemical knockdown strategies utilize bifunctional molecules to activate the protein quality control system. In these methods, a typical bifunctional molecule contains two fragments, the recognition fragment that

${ }^{a}$ Key Laboratory of Bioorganic Phosphorus Chemistry and Chemical Biology (Ministry of Education), Department of Chemistry, Tsinghua University, Beijing 100084, China. E-mail: liym@mail.tsinghua.edu.cn; Fax: +86-10-62781695

${ }^{b}$ Beijing Institute for Brain Disorders, Beijing 100069, China

${ }^{c}$ Key Laboratory of Modern Toxicology of Shenzhen, Shenzhen Center for Disease Control and Prevention, Shenzhen 518055, China

$\dagger$ Electronic supplementary information (ESI) available. See DOI: 10.1039/c7ra05347a can specifically identify the target proteins and the activation fragment that can stimulate the protein quality control system. Various approaches have been developed based on chemical knockdown strategies such as the method of proteolysis targeting chimeras (PROTACs), ${ }^{10}$ the auxin-inducible degron (AID), ${ }^{11}{\text { Boc } 3 \mathrm{Arg}^{12}}^{12}$ and phthalimide conjugations ${ }^{13}$ and hydrophobic tagging. ${ }^{14}$ These approaches have been successfully applied to degrade many important proteins. ${ }^{15}$

Studies showed that the hydrophobic group of the misfolded protein is exposed to its surface, which can be recognized and degraded by the ubiquitin-proteasome system (UPS). ${ }^{12}$ Based on the mechanism that the protein attached with a hydrophobic tag can mimic the misfolded protein, Crews and his coworkers developed a novel method called 'hydrophobic tagging (HyT)' ${ }^{14}$ The hydrophobic group can be linked to the HaloTag fusion protein to increase its hydrophobicity and thus facilitating its clearance by UPS. ${ }^{14}$ Furthermore, it was found that molecules containing the hydrophobic group and the target protein ligand could induce the target protein degradation. ${ }^{16}$ So we can control the level of the protein by the HyT method. ${ }^{14,16} \mathrm{HyT}$ is suitable for any proteins, since it just requires the addition of a low-molecular-weight hydrophobic group to the target proteins..$^{14,16,17 a, b}$

In our previous work, we utilized the PROTACs method to effectively reduce Tau level. ${ }^{15 d}$ The PROTACs and HyT methods induced the protein degradation by the same cellular quality control system without any genetic manipulation. And these two techniques need little or no synthetic chemistry. Recently, these two methods have been employed to degrade endogenous proteins successfully. HyT has similar advantages to PROTACs such as universality and potent degradation effect. Here, we want to develop diversity methods to mediated Tau level. In this work, we used the HyT method to regulate the level of Tau in vitro and in vivo. Furthermore, compared to our previous work, our current results showed that the HyT method was more 
effective on Tau degradation in animal experiments. To regulate the level of Tau specifically, we designed a tri-functional molecule, HyT-Tau-CPP. The HyT-Tau-CPP contains three motifs: 'hydrophobic tag motif', 'Tau-recognition motif' and 'cellpenetrating peptide motif' (Fig. 1A). The Tau-recognition motif is the C-terminal regions of tubulin subunits sequences YQQYQDATADEQG ${ }^{\mathbf{1 8}}$ that can specifically bind to Tau. We used adamantly as the hydrophobic tag motif because it had some advantages over some other molecules such as small molecular weight and strong hydrophobicity. Moreover, it has been demonstrated that adamantyl could mediate the degradation of many different proteins. ${ }^{\mathbf{1 4 1 6 , 1 7 a , b}}$ We chose ploy-D-arginine

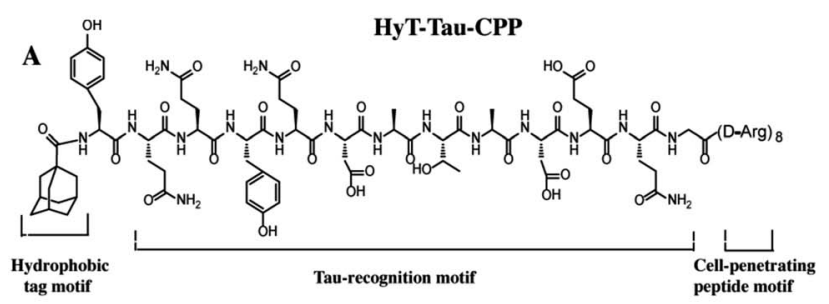

B

CF-HyT-Tau-CPP
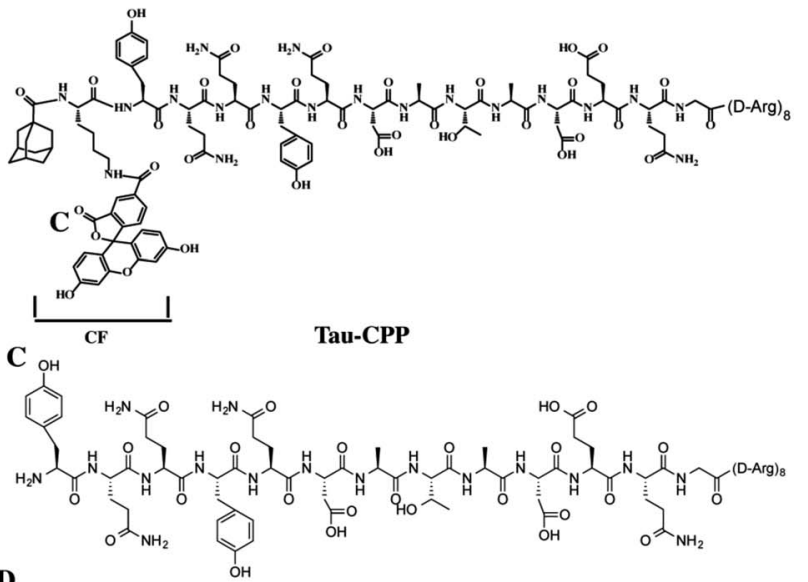

D

\section{HyT-MTau-CPP}

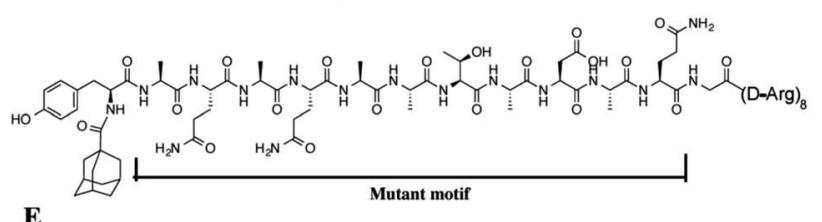

$\mathbf{E}$

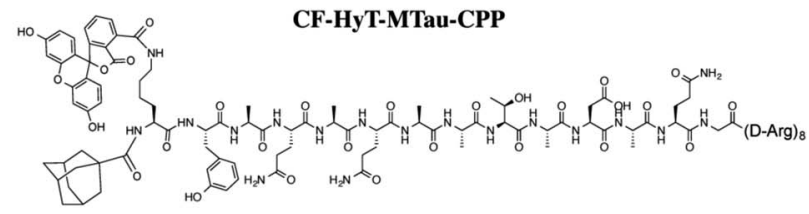

Fig. 1 (A) Structure of HyT-Tau-CPP. It consists of three motifs: a hydrophobic tag. Tau recognition motif and cell-penetrating peptide. (B) Structure of CF-HyT-Tau-CPP. Adding a fluorescence tag to HyT-Tau-CPP. (C) Structure of Tau-CPP without a hydrophobic tag. (D) Structure of HyT-MTau-CPP. The Tau recognition motif YQQYQDATADEQG was mutated to YYQAQAATADAQG. (E) Structure of CF-HyT-MTau-CPP. Adding a fluorescence tag to HyT-MTau-CPP. We synthesized all peptides by solid-phase peptide synthesis method.
(D-Arg) $)_{8}$ (ref. 10c) as the cell-penetrating peptide (CPP) motif since it was used widely in many fields. We synthesized HyT-TauCPP by solid-phase peptide synthesis method (ESI Fig. S1†). We supposed that HyT-Tau-CPP could penetrate cell membranes, specifically recognize and degrade Tau in vitro and in vivo.

Firstly, to evaluate the binding affinity between recombinant hTau40 protein and HyT-Tau-CPP in vitro, we appended the 5(6)-carboxyfluorescein (CF) to the N-terminus of HyT-Tau-CPP and HyT-MTau-CPP to get 'CF-HyT-Tau-CPP' (Fig. 1B) and 'CFHyT-MTau-CPP' (Fig. 1E). Recombinant hTau40 protein was expressed by Escherichia coli, BL21 strain (ESI Fig. S2A $\dagger$ ). Then the fluorescence polarization assay was applied. The fluorescence polarization results showed that CF-HyT-Tau-CPP could bind to Tau specifically $\left(K_{\mathrm{d}}=0.7655 \pm 0.1882 \mu \mathrm{M}\right)$ and the 'HyTMTau-CPP' didn't have the ability to bind to Tau (Fig. 2A). $\mathrm{CF}$ also didn't bind to Tau (ESI Fig. S2B $\dagger$ ). Moreover, to investigate whether HyT-Tau-CPP could penetrate into cells, we incubated $150 \mu \mathrm{M}$ CF-HyT-Tau-CPP with the wild type N2a cells for $6 \mathrm{~h}$. The laser scanning confocal microscope was applied and the confocal microscope results indicated that CF-HyT-TauCPP had a high ability to get into cells in a short time, and evenly distributed in the cytoplasm (Fig. 2B and ESI Fig. S3†). In addition, the wild type N2a cells were incubated with $150 \mu \mathrm{M}$ CF-HyT-Tau-CPP for 0 h, 1 h, 3 h, 4 h, 6 h and 8 h respectively. Flow cytometry was used to detect the fluorescence intensity changes in cells. The results showed that the fluorescence intensity increased gradually (Fig. 2C). Together, these results
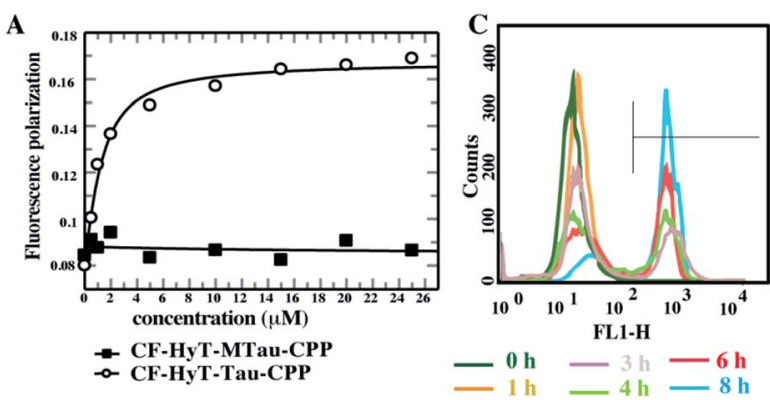

B

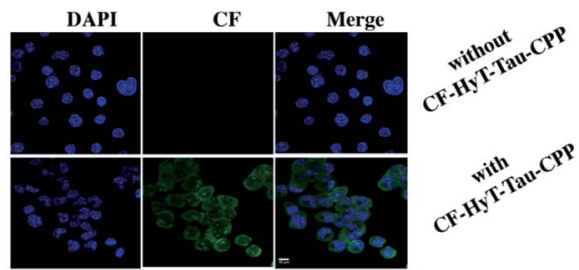

Fig. 2 HyT-Tau-CPP is specific for Tau in vitro and can penetrate into cells in a short time. (A) The fluorescence polarization results of $0 \mu \mathrm{M}$, $0.5 \mu \mathrm{M}, 1 \mu \mathrm{M}, 2 \mu \mathrm{M}, 5 \mu \mathrm{M}, 10 \mu \mathrm{M}, 15 \mu \mathrm{M}, 20 \mu \mathrm{M}, 25 \mu \mathrm{M}$ Tau 441 protein with $0.1 \mu \mathrm{M}$ CF-HyT-Tau-CPP and $0.1 \mu \mathrm{M}$ CF-HyT-MTau-CPP respectively. Circles represent CF-HyT-Tau-CPP and squares represent CF-HyT-MTau-CPP. (B) The confocal microscope results of the wild type N2a cells with the treatment of $150 \mu \mathrm{M}$ CF-HyT-Tau-CPP for 6 h. Blue, nucleus; green, CF-HyT-Tau-CPP; scale bar, $10 \mu \mathrm{m}$. (C) Entry of CF-HyT-Tau-CPP into wild-type N2a cells tested by flow cytometry. The gray horizontal line represents the fluorescence positive signal threshold. 
A

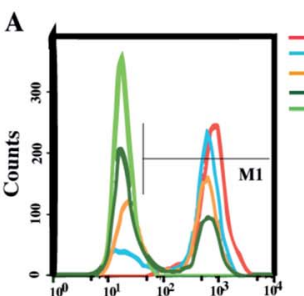

FL1-H
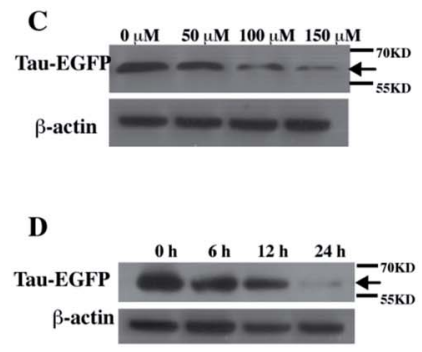

$\mathbf{E}$

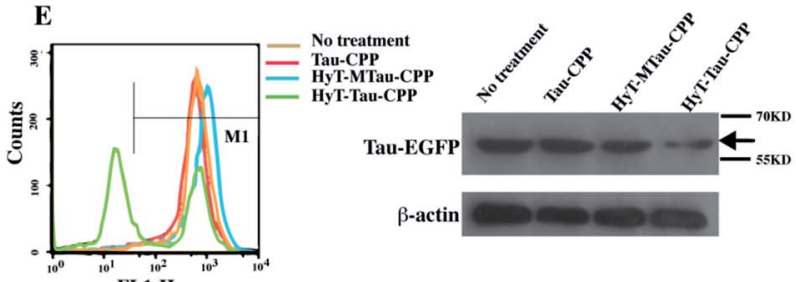

B
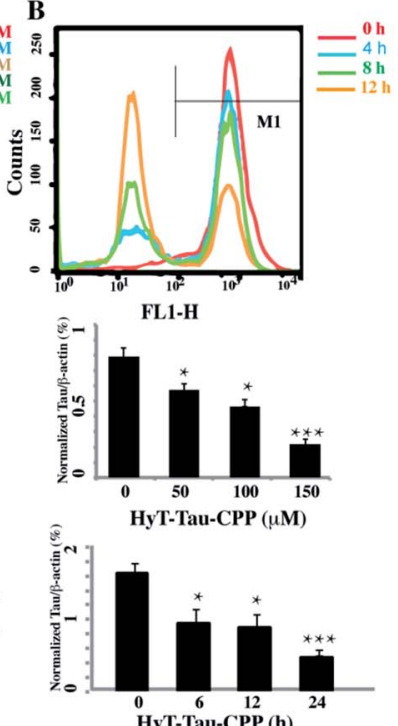

HyT-Tau-CPP(h)
Fig. 3 HyT-Tau-CPP can lead to the degradation of intracellular Tau effectively. (A) The flow cytometry results of the Tau-EGFP overexpressed cells with the treatment of $0 \mu \mathrm{M}, 50 \mu \mathrm{M}, 100 \mu \mathrm{M}, 150 \mu \mathrm{M}$, $200 \mu \mathrm{M}$ of HyT-Tau-CPP for $24 \mathrm{~h}$. The gray horizontal line represents the fluorescence positive signal threshold. (B) The flow cytometry results of the Tau-EGFP overexpressed cells with the treatment of $150 \mu \mathrm{M}$ HyT-Tau-CPP for different time. The gray horizontal line represents the fluorescence positive signal threshold. (C) Representative western blot results for Tau and $\beta$-actin revealed a concentration-dependent degradation of Tau treated with $0 \mu \mathrm{M}, 50 \mu \mathrm{M}, 100 \mu \mathrm{M}$, $150 \mu \mathrm{M}$ HyT-Tau-CPP for $24 \mathrm{~h}$. Values for all Tau and $\beta$-actin are mean \pm SD ( $n=3$ independent experiments). The level of Tau is measured by densitometry and normalized with $\beta$-actin level. Statistical significance compared to the control $(0 \mu \mathrm{M})$ was analyzed by oneway ANOVA, $* P<0.05$. ${ }^{*} * P<0.01$. $* * * P<0.001$. (D) Representative western blot results for Tau and $\beta$-actin revealed a time-dependent knockdown of Tau with the treatment of $150 \mu \mathrm{M}$ HyT-Tau-CPP for $0 \mathrm{~h}$, $6 \mathrm{~h}, 12 \mathrm{~h}, 24 \mathrm{~h}$ in the same cell line. Values for all Tau and $\beta$-actin are mean \pm SD ( $n=3$ independent experiments). The level of Tau is measured by densitometry and normalized with $\beta$-actin level. Statistical significance compared to the control $(0 \mathrm{~h})$ was analyzed by oneway ANOVA, $* P<0.05$. $* * P<0.01$. $* * * P<0.001$. (E) The flow cytometry and western blot for Tau and $\beta$-actin results of the TauEGFP overexpressed cells with the treatment of $150 \mu \mathrm{M}$ Tau-CPP, 150 $\mu \mathrm{M}$ HyT-MTau-CPP, $150 \mu \mathrm{M}$ HyT-Tau-CPP or vector for $24 \mathrm{~h}$. The results demonstrated that the Tau-CPP and HyT-MTau-CPP couldn't demonstrated that HyT-Tau-CPP could interact with Tau specifically in vitro and cross the cell membranes.

We next generated a Tau-EGFP overexpressed cell line based on N2a to investigate whether HyT-Tau-CPP could degrade intracellular Tau in cell culture. The Tau-EGFP overexpressed cells were firstly treated with a protein biosynthesis inhibitor, cycloheximide (CHX, $10 \mu \mathrm{g} \mathrm{ml}^{-1}$ ) for $2 \mathrm{~h}$. Then we incubated the Tau-EGFP overexpressed cells with increasing concentrations of HyT-Tau-CPP for $24 \mathrm{~h}$ and $150 \mu \mathrm{M}$ HyT-Tau-CPP in different time respectively. To assess the degradation extent of HyT-TauCPP-mediated, we analyzed Tau level by the flow cytometry assay and western blot. The flow cytometry results showed that the intracellular fluorescence intensity reduced in a concentration-dependent and time-dependent manners, which demonstrated that HyT-Tau-CPP could decrease tau level in cells (Fig. 3A and B; quantified in ESI Fig. S4A and B $†$ ). The results of western blot also showed significant decline of intracellular Tau level (Fig. 3C and D; quantified in ESI Fig. S4C and D; ESI Fig. S5A-D $\dagger$ ). The maximal degradation level was approximately $80 \%$ in a dose-dependent manner and $75 \%$ in time-course experiment (Fig. 3C and D). In addition, to verify the degradation effect on the endogenous Tau, the wild type N2a cells were incubated with $150 \mu \mathrm{M}$ HyT-Tau-CPP or vector for $24 \mathrm{~h}$. The western blot results showed that HyT-Tau-CPP could also reduce endogenous Tau levels (ESI Fig. S4E $\dagger$ ). To confirm the specificity of the Tau recognition motif and the necessity of the adamantyl, we synthesized two control peptides: 'Tau-CPP' that didn't have adamantly group (Fig. 1C) and 'HyT-MTau-CPP' whose 'Tau-recognition motif' YQQYQDATADEQG was mutated to YYQAQAATADAQG (Fig. 1D). The western blot results showed that the 'Tau-CPP' and 'HyT-MTau-CPP' didn't have the ability to induce the degradation of Tau (Fig. 3E). To further confirm the degradation effect of HyT-Tau-CPP, we observed the Tau level by the laser scanning confocal microscope. The EGFP fluorescence intensity decreased significantly (Fig. 3F). Again, the results revealed the efficient degradation effect of HyT-TauCPP. Together, these data provide experimental evidence that HyT-Tau-CPP is able to dose- and time-dependently downregulate the Tau level in cell culture.

To confirm that HyT-Tau-CPP-mediated Tau degradation was the proteasome dependent, we incubated the Tau-EGFP overexpressed cells with $150 \mu \mathrm{M}$ HyT-Tau-CPP for $18 \mathrm{~h}$. The cells were also treated with $10 \mu \mathrm{M}$ proteasome inhibitor MG132 for $6 \mathrm{~h}$ before harvest. Tau levels were analyzed by western blot assay. The results showed that the proteasome inhibitors MG132 blocked HyT-Tau-CPP-mediated degradation, which indicated that HyT-Tau-CPP-mediated Tau degradation depended on the proteasome (Fig. 4A and ESI Fig. S5E-I†).

To evaluate the degree of HyT-Tau-CPP-mediated cytotoxicity, a 3-(4,5-dimethyl-2-thiazoyl)-2,5-diphenyl tetrazolium

induce the degradation of Tau. The gray horizontal line in flow cytometry is on behalf of the fluorescence positive signal threshold. (F) Confocal microscopic images analysis showed the level of Tau-EGFP in the same cell line treated with $100 \mu \mathrm{M}, 150 \mu \mathrm{M}$ or without HyT-TauCPP for $24 \mathrm{~h}$. Blue, DAPI-stained nucleus; green, EGFP-Tau; scale bar, $10 \mu \mathrm{m}$. Full blot images for $C$ and $D$ are shown in ESI Fig. S5. $\dagger$ 
A

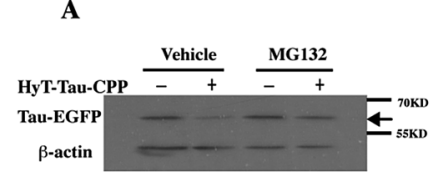

B

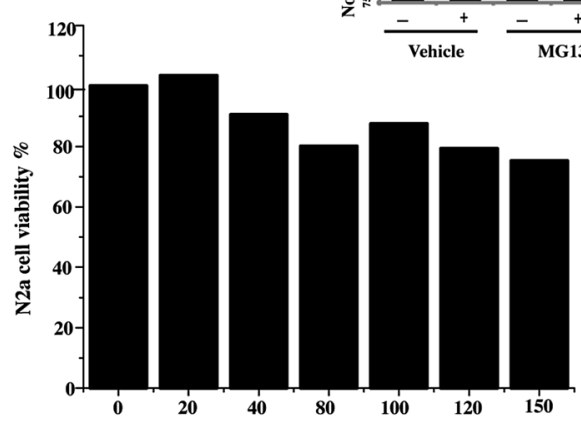

C

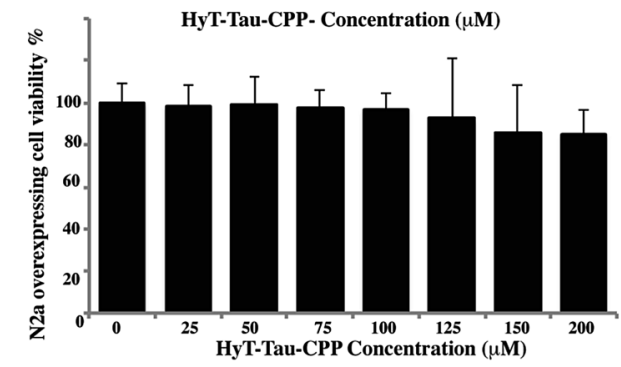

Fig. 4 HyT-Tau-CPP-induced degradation is mediated by the proteasome with slight cytotoxicity. (A) Representative western blot results for Tau and $\beta$-actin of the Tau-EGFP overexpressed cells with the treatment of $10 \mu \mathrm{M}$ MG132 as indicated. $\beta$-actin acted as a loading control. Values for all Tau and $\beta$-actin are mean \pm SD $(n=3$ independent experiments). The level of Tau is measured by densitometry and normalized with $\beta$-actin level. Statistical significance was analyzed by one-way ANOVA, $* P<0.05$. $* * P<0.01$. (B) Cell viability results of the wild type N2a cells treated with $0 \mu \mathrm{M}, 20 \mu \mathrm{M} 40 \mu \mathrm{M}, 80 \mu \mathrm{M}$, $100 \mu \mathrm{M}, 120 \mu \mathrm{M}, 150 \mu \mathrm{M}$ HyT-Tau-CPP. Data are detected by MTT assay. Vales are means $\pm S D, n=5$. (C) Cell viability results of the Tau-EGFP overexpressed cells treated with $0 \mu \mathrm{M}, 25 \mu \mathrm{M}, 50 \mu \mathrm{M}, 75 \mu \mathrm{M}$, $100 \mu \mathrm{M}, 125 \mu \mathrm{M}, 150 \mu \mathrm{M}, 200 \mu \mathrm{M}$ HyT-Tau-CPP. Data are detected by MTT assay. Vales are means $\pm S D, n=5$.

bromide (MTT) assay was applied to measure the cell survival. Wild type N2a cells were treated with different concentration of HyT-Tau-CPP for $24 \mathrm{~h}$. The quantification of cell survival showed that the cell survival was about $80 \%$ when the cells were incubated with $150 \mu \mathrm{M}$ HyT-Tau-CPP. A relatively small cellular toxicity was observed below the concentration of $200 \mu \mathrm{M}$ (Fig. 4B). Furthermore, the overexpressed cells were also treated with different concentration of HyT-Tau-CPP for $24 \mathrm{~h}$. Similarly, HyT-Tau-CPP also had a little cytotoxicity to the overexpressed cells (Fig. 4C).

We further verified the degradation effect of HyT-Tau-CPP in vivo. An AD mouse model (B6, 3xTg-AD mice, 5 to 6 months old, 129-Psen1tm1Mpm Tg [APPSwe, Tau P301L]1Lfa/Mmjax) ${ }^{15 d}$ was treated with HyT-Tau-CPP (18 $\mathrm{mg} \mathrm{kg}^{-1}$ body weight, $n=4$ for each group) with intravenous injection, each mouse was treated with HyT-Tau-CPP for 8 days. The western blot data showed that HyT-Tau-CPP could reduce the level of Tau in the hippocampus and cerebral cortex regions of $\mathrm{AD}$ mouse models significantly
(Fig. 5A and B and ESI Fig. S6 $\dagger$ ). In addition, immunohistochemistry results were similar to the western blot results (Fig. 5C).

Recently, it was also reported that Tau reduction could rescue $A \beta$-induced damages in axonal transport by gene knockout. Therefore, Tau reduction may become a potential strategy for AD treatment. However, Tau protein also played a key role in brain, and tau deficiency was previously showed to cause neurodegeneration. ${ }^{19 a-e}$ Here, we just supplied a novel chemical method to regulate total Tau levels and our results provided a good basis for further research. For clinical application, there is still much work to do due to potential caveats of using Tau reduction strategy.

In this work, we designed and synthesized a bifunctional peptide HyT-Tau-CPP based on the HyT method. We found that HyT-Tau-CPP was highly specific for Tau in vitro and could get into cells in a short time. HyT-Tau-CPP could also lead to significant degradation of endogenous Tau with slight cellular toxicity. Furthermore, it could lower the Tau level in 3xTg-AD
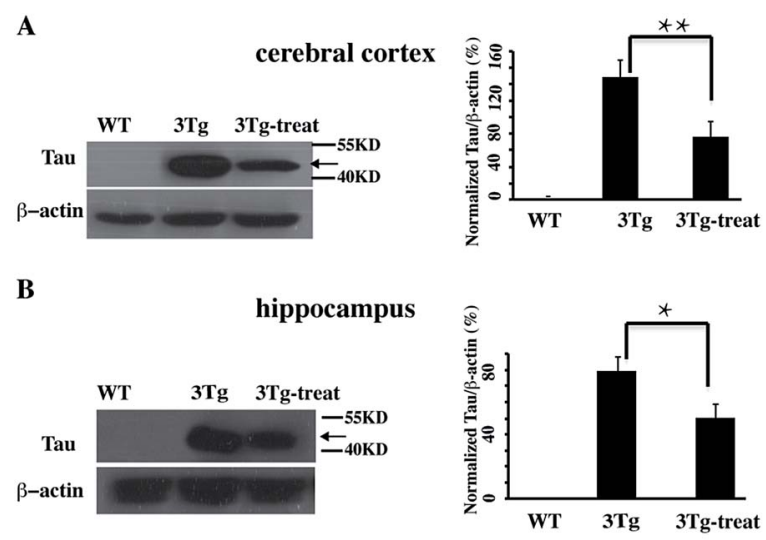

C
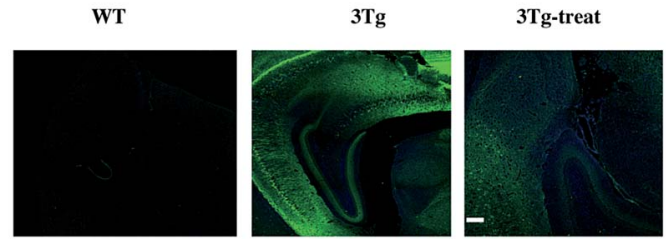

Fig. 5 HyT-Tau-CPP can reduce the Tau level in AD model mice. (A) Representative western blot results of Tau in the brain cerebral cortex in transgenic mice with the treatment of HyT-Tau-CPP (B6, 3xTg-AD mice, 5 to 6 months old, 129-Psen1tm1Mpm Tg [APPSwe, Tau P301L] $1 \mathrm{Lfa} /$ Mmjax). B6129SF2/J mice were used as the controls. Values for all Tau and $\beta$-actin are mean \pm SD ( $n=3$ independent experiments). The level of Tau is measured by densitometry and normalized with $\beta$-actin level. Statistical significance was analyzed by one-way ANOVA, $* P<$ 0.05. ${ }^{*} P<<0.01$. (B) Representative western blot results of Tau level of the brain hippocampus in the same transgenic mice as (A). Values for all Tau and $\beta$-actin are mean \pm SD ( $n=3$ independent experiments). The level of Tau is measured by densitometry and normalized with $\beta$ actin level. Statistical significance was analyzed by one-way ANOVA, $* P<0.05$. (C) Immunohistochemistry results of Tau level of the brain cerebral cortex and hippocampus in transgenic mice with the treatment of HyT-Tau-CPP in the same animal model. Tau was probed with anti-tau antibody and the secondary antibody is donkey anti-rabbit IgG $(\mathrm{H}+\mathrm{G})$ antibody conjugated with Alexa Fluor 488. Scale bar, $50 \mu \mathrm{m}$. Full blot images for $A$ and $B$ are shown in ESI Fig. S6.† 
mice. We also confirmed that HyT-Tau-CPP-mediated Tau degradation was proteasome dependent. In summary, we applied the HyT strategy to induce the degradation of $\mathrm{AD}$ related Tau both in vitro and in vivo for the first time. Importantly, HyTTau-CPP may become a potential therapeutic strategy in the treatment of $\mathrm{AD}$.

\section{Ethical conduct of animal experiments}

All experiments with animals were performed in compliance with the relevant laws and Chinese institutional guidelines for animal experiments. All animals used in this study were treated in accordance with the Institutional Animal Care and Use Committee (IACUC) and the Laboratory Animal Facility of Tsinghua University, and approved by the Institutional Animal Care and the Laboratory Animal Facility of Tsinghua University.

\section{Conflicts of interest}

The authors declare that there are no conflicts of interest.

\section{Author contributions}

Na Gao did most of the experiments, analyzed the data, and wrote the paper. Ting-Ting Chu and Qian-Qian Li constructed the overexpressing cell line. Yeh-Jun Lim and Tian Qiu did some animal experiments. X.-F. Y. provided the mouse model. MengRong Ma, Zhi-Wen $\mathrm{Hu}$, and Yu-Fen Zhao discussed the work and analyzed the data. Yong-Xiang Chen and Yan-Mei Li designed the experiments, analyzed the data, and wrote the paper.

\section{Acknowledgements}

This work was supported by the Major State Basic Research Development Program of China (2013CB910700) and the National Natural Science Foundation of China (21472109, 81661148047).

\section{References}

1 C. Ballatore, V. M. Y. Lee and J. Q. Trojanowski, Nat. Rev. Neurosci., 2007, 8, 663-672.

2 J. Di, L. S. Cohen and C. P. Corbo, Sci. Rep., 2016, 6, 20833, DOI: $10.1038 /$ srep20833.

3 (a) M. Rapoport, H. N. Dawson and L. I. Binder, Proc. Natl. Acad. Sci. U. S. A., 2002, 99, 6364-6369; (b) K. A. Vossel, K. Zhang and J. Brodbeck, Science, 2010, 330, 198; (c) E. D. Roberson, K. Scearce-Levie and J. J. Palop, Science, 2007, 316, 750-754.

4 (a) E. Giacobini and G. Gold, Nat. Rev. Neurol., 2013, 9, 677686; (b) Z. Liu, A. Zhang and H. Sun, RSC Adv., 2017, 7, 60466058; (c) K. R. Brunden, J. Q. Trojanowski and V. M. Y. Lee, Nat. Rev. Drug Discovery, 2009, 8, 783-793; (d) W. H. Wu, P. Lei, Q. Liu, J. Hu, A. P. Gunn, M. S. Chen, Y. F. Rui, X. Y. Su, Z. P. Xie, Y. F. Zhao, A. I. Bush and Y. M. Li, J. Biol. Chem., 2008, 283, 31657-31664; (e) T. T. Chu, Q. Q. Li, T. Qiu, Z. Y. Sun, Z. W. Hu, Y. X. Chen, Y. F. Zhao and
Y. M. Li, Mol. BioSyst., 2014, 10, 3081-3085; (f) U. Krüger, Y. Wang and S. Kumar, Neurobiol. Aging, 2012, 33, 22912305.

5 F. H. Bouwman, N. S. M. Schoonenboom and N. A. Verwey, Neurobiol. Aging, 2009, 30, 1895-1901.

6 L. M. Ittner, Y. D. Ke and F. Delerue, Cell, 2010, 142, 387-397.

7 P. Tompa, Trends Biochem. Sci., 2002, 27, 527-533.

8 D. Bumcrot, M. Manoharan, V. Koteliansky and D. W. Sah, Nat. Chem. Biol., 2006, 2, 711-719.

9 D. L. Buckley and C. M. Crews, Angew. Chem., Int. Ed., 2014, 53, 2312-2330.

10 (a) K. M. Sakamoto, Proc. Natl. Acad. Sci. U. S. A., 2001, 98, 8554-8559; (b) A. R. Schneekloth, M. Pucheault, H. S. Tae and C. M. Crews, Bioorg. Med. Chem. Lett., 2008, 18, 59045908; (c) J. S. Schneekloth, J. Am. Chem. Soc., 2004, 126, 3748-3754; (d) J. Hines, J. D. Gough, T. W. Corson and C. M. Crews, Proc. Natl. Acad. Sci. U. S. A., 2013, 110, 89428947.

11 A. J. Holland, D. Fachinetti and J. S. Han, Proc. Natl. Acad. Sci. U. S. A., 2012, 109, 3350-3357.

12 (a) M. J. C. Long, D. R. Gollapalli and L. Hedstrom, Chem. Biol., 2012, 19, 629-637; (b) T. K. Neklesa and C. M. Crews, Nature, 2012, 487, 308-309.

13 (a) G. E. Winter, D. L. Buckley, J. Paulk, J. M. Roberts, A. Souza, S. Dhe-Paganon and J. E. Bradner, Science, 2015, 348, 1376-1381; (b) J. Kronke, E. C. Fink, P. W. Hollenbach, K. J. MacBeth, S. N. Hurst, N. D. Udeshi, P. P. Chamberlain, D. R. Mani, H. W. Man and A. K. Gandhi, Nature, 2015, 523, 183-188.

14 T. K. Neklesa, H. S. Tae, A. R. Schneekloth, M. J. Stulberg, T. W. Corson, T. B. Sundberg, K. Raina, S. A. Holley and C. M. Crews, Nat. Chem. Biol., 2011, 7, 538-543.

15 (a) M. Zengerle, K. H. Chan and A. Ciulli, ACS Chem. Biol., 2015, 10, 1770-1777; (b) D. Remillard, D. L. Buckley and J. Paulk, Angew. Chem., Int. Ed., 2017, 56, 1-7; (c) D. P. Bondeson, A. Mares, I. E. Smith, J. D. Harling, I. Churcher and C. M. Crews, Nat. Chem. Biol., 2015, 11, 611-617; (d) T. T. Chu, N. Gao, Q. Q. Li, P. G. Chen, X. F. Yang, Y. X. Chen, Y. F. Zhao and Y. M. Li, Cell Chem. Biol., 2016, 23, 453-461.

16 (a) J. L. Gustafson, T. K. Neklesa and C. S. Cox, Angew. Chem., Int. Ed., 2015, 127, 9795-9798; (b) S. M. Lim, T. Xie and K. D. Westover, Bioorg. Med. Chem. Lett., 2015, 25, 33823389.

17 (a) T. Xie, S. M. Lim, K. D. Westover, M. E. Dodge, P. A. Janne, C. M. Crews and N. S. Gray, Nat. Chem. Biol., 2014, 10, 10061012; (b) K. Raina, D. J. Noblin, Y. V. Serebrenik, A. Adams, C. Zhao and C. M. Crews, Nat. Chem. Biol., 2014, 10, 957-962. 18 C. I. Rivas, J. C. Vera and R. B. Maccioni, Proc. Natl. Acad. Sci. U. S. A., 1988, 85, 6092-6096.

19 (a) P. Lei, S. Ayton and S. Moon, Mol. Neurodegener., 2014, 9, 29; (b) P. Lei, S. Ayton and D. I. Finkelstein, Nat. Med., 2012, 18, 291-295; (c) P. Lei, S. Ayton and A. T. Appukuttan, Mol. Psychiatry, 2017, 22, 396-406; (d) M. Zheng, L. Jiao and X. Tang, FASEB J., 2017, 31, 3349-3358; (e) Q. L. Ma, X. Zuo and F. Yang, J. Neurosci., 2014, 34, 7124-7136. 